\title{
Uterus transplantation in a sheep model: novel surgical technique with long-term survival and uterus vitality. First case series in Argentina
}

\author{
Martín A Maraschio, José M Sad Larcher², Alvaro Alcaraz¹, Enzo Giordano ${ }^{1}$, Santiago Reimondez¹, Oscar Luján ${ }^{3}$, \\ Marcelo Iraci ${ }^{4}$, José L Sereno ${ }^{5}$, Analía Priotto ${ }^{6}$, Olga Domínguez ${ }^{1}$, Pablo Valle ${ }^{7}$, Maximiliano Abrego ${ }^{2}$, Soledad \\ Rubio $^{8}$, Cristian Lamberto ${ }^{9}$, Eduardo Villada ${ }^{10}$ \\ ${ }^{1}$ Liver, Kidney and Pancreas Transplant Program in Hospital Privado Universitario de Córdoba, Argentina \\ ${ }^{2}$ Department of Obstetrics and Gynecology of Hospital Privado Universitario de Córdoba, Argentina \\ ${ }^{3}$ Department of Animal Clinic, Faculty of Agronomy and Veterinary, Universidad Nacional de Río Cuarto, Córdoba, \\ Argentina \\ ${ }^{4}$ Department of Surgical Pathologies, Faculty of Agronomy and Veterinary, Universidad Nacional de Río Cuarto, \\ Córdoba, Argentina \\ ${ }^{5}$ Hospital Escuela of the Faculty of Agronomy and Veterinary, Universidad Nacional de Río Cuarto, Córdoba, \\ Argentina \\ ${ }^{6}$ Department of Anesthesiology, Hospital Privado Universitario de Córdoba, Argentina \\ ${ }^{7}$ Department of Orthopedics and Traumatology, Hospital Privado Universitario de Córdoba, Argentina \\ ${ }^{8}$ Department of Plastic and Reconstructive Surgery, Hospital Privado Universitario de Córdoba, Argentina \\ ${ }^{9}$ Imaging Department, Sanagec Clinic, Córdoba, Argentina \\ ${ }^{10}$ Veterinary Hospital, Universidad Católica de Córdoba, Argentina
}

\begin{abstract}
Objective: To develop a sheep autologous uterus transplantation (UT) program with an innovative surgical technique and assess long term uterus vitality and animal survival.

Methods: A novel surgical technique consisting of the procurement of the complete uterus and the two ovaries, back table vascular reconstruction, and subsequent implantation in the same animal, performing only two arterial and two venous anastomoses.

Results: Four autologous transplantations were performed; anesthesia and surgery were well tolerated by all the animals without complications. Direct observation and Doppler US performed a week after UT and laparoscopy performed three months later confirmed uterus vitality. All animals were alive more than a year after transplantation.

Conclusions: Our study was the first to describe a novel surgical technique for sheep uterus autologous transplantation in Latin America, showing long-term survival and uterus vitality.
\end{abstract}

Keywords: uterus transplantation, infertility, female surgery, animals

\section{INTRODUCTION}

Absolute uterine factor infertility (AUFI) remains as one of the few types of infertility without treatment. The only options for women with AUFI until a few years ago were adoption or surrogate pregnancy (Beski et al., 2000; Friedler et al., 2016; Brännström, 2017; Dahm-Kähler et al., 2016). For these patients, uterus transplantation (UT) has become an effective therapy, allowing them to carry out an entire pregnancy and give birth (Brännström et al., 2015).

Since the first successful live birth from a UT recipient reported by Brännstrom and colleagues in 2014 (Brännström et al., 2014; 2015), more than 70 UT procedures have been reported, with more than 20 live births worldwide and a rapidly increasing number of procedures including surgical techniques, immunosuppression protocols, donor and recipient selection criteria, and other matters associated with UT (Brännström et al., 2019; Brännström, 2020; Castellón et al., 2017). Nevertheless, caution must be taken until sufficient experience has been gained and the therapy has evolved from an experimental procedure to a safe and effective technique that can be broadly reproduced (Daolio et al., 2020; Riggan et al., 2020; Practice Committee of the American Society for Reproductive Medicine, 2018; Farrell \& Falcone, 2015)

Given the lack of transplant centers with significant experience on the procedure and the limits to acquiring experience performing UT in humans, animal experimentation has emerged as an important surgical training tool (Brännström et al., 2012; Racho El-Akouri et al., 2003; Andraus et al., 2017; Ramirez et al., 2008; Wei et al., 2013; Wranning et al., 2008).

This report presents our experience with sheep autologous UT of the entire uterus and the two ovaries with back table vascular reconstruction, with successful reperfusion achieved with only two arterial and two venous anastomoses.

\section{OBJECTIVE}

To develop a sheep autologous uterus transplantation (UT) program with an innovative surgical technique and assess long term uterus vitality and animal survival.

\section{MATERIAL AND METHODS}

Mature Hampshire Down ewes (2-3 years of age; 50-70 $\mathrm{kg}$ ) with proven fertility (previous pregnancy leading to live birth) screened for infectious diseases (Brucellosis, Clostridium, ecto and endoparasites) were purchased from an accredited breeder. The animals arrived at the Animal Hospital of the Universidad Católica de Córdoba three weeks before surgery and were kept in a controlled 12/12 h light/dark cycle. The animals had free access to hay and water twice a day. The study followed the guidelines for handling and care of experimental animals and was approved by the Commission of Animal Welfare of the Universidad Católica de Córdoba. 


\section{Anesthesia}

Food was withdrawn 24 hours before surgery. Intramuscular xylazine $2 \%$ at 0.5 to $1 \mathrm{mg} / \mathrm{kg}$ was administered as a pre-anesthetic drug 20 minutes before the animals were taken to the operating room; cotton plugs were placed in their ears to prevent animal stress. An external jugular vein access was performed. Intravenous propofol was used for anesthesia induction $(1 \mathrm{mg} / \mathrm{kg} / \mathrm{min})$ until mastication muscles relaxed and palpebral reflex was lost. Endotracheal intubation was performed with the sheep in full neck extension in a ventral supine position; the breathing tube was secured to the animal jaw. The eyeballs were covered but maintained accessible for clinical assessment of correct anesthetic plane. An orogastric tube was placed to minimize esophageal reflux. Esophageal temperature was monitored, as well as blood pressure and pulse oximetry.

Anesthesia was maintained with isoflurane and fentanyl $(10 \mathrm{mcg} / \mathrm{Kg} / \mathrm{h})$; noradrenaline was used to maintain MAP over $65 \mathrm{~mm} / \mathrm{Hg}$ and the heart rate at 80 to $95 \mathrm{bpm}$.

Ventilation was administered in spontaneous mode, keeping breathing rate and depth on the upper normal range to avoid respiratory acidosis. Once surgery was concluded, 2.5 $\mathrm{ml}$ of intramuscular neostigmine methyl sulfate $(200 \mathrm{mg} / 100$ $\mathrm{mL}$ ) combined with diphenhydramine hydrochloride $(2 \mathrm{~g} / 100$ $\mathrm{mL}$ ) were administered to help recover rumen movements and maintained in a daily basis for three consecutive days, with frequent assessment of rumen movements. Intramuscular flunixin meglumine was given for pain relief. Antibiotic prophylaxis was administered and then maintained for five days with enrofloxacin. Once the swallowing reflex was recovered, the animals were fed with alfalfa hay, avoiding grains.

\section{Surgery}

With the animal fully anesthetized in dorsal decubitus, the legs were restrained to the operating table; the abdominal area was shaved and thoroughly scrubbed with soapy antiseptic to remove skin lanolin. The abdomen and vagina were prepped with iodized solution, and the abdomen was accessed with a broad midline laparotomy, taking care not to cut through the mammary vein. The cavity of the rumen was drained with a tube to allow better abdominal exposure. The uterus, the two ovaries, and the fallopian tubes (adnexa) were carefully dissected including the ovarian veins and the uterine and ovarian arteries up to the internal iliac artery and aorta, respectively. External iliac vessels were dissected as well, for later vascular anastomosis.

The uterus was removed; the ovarian arteries with aortic patches were repaired with a patch from the internal iliac artery, and the uterine arteries with a portion of approximately $5-10 \mathrm{~mm}$ of the cephalic and caudal portions of the first part of the internal iliac arteries (Fig. 1). The uterine veins were sectioned at the level of the iliac veins. A portion of the upper vagina was resected along with the uterus. The round ligaments were preserved with the uterus, since they would be later used for organ fixation after re-implantation. Cold ischemic time was set at the moment of uterus extraction from the abdominal cavity.

For bench surgery, the uterus was positioned on a recipient with sterile ice and perfused through uterine and ovarian arteries with Histidine-Tryptophan-Ketoglutarate $\AA$ organ preservation solution (HTK, Dr. Franz Köhler Chemie $\mathrm{GmbH}$, Bensheim, Germany) at $4^{\circ} \mathrm{C}$ with sufficient pressure until vein outflow turned clear and the uterus lost color uniformly. The distal end of the internal iliac artery (from which the uterine artery emerged) was anastomosed to the aortic patch (to which the ovarian arteries continued) with a running 8-0 or 9-0 polypropylene under $6 x$ loupe magnification with microsurgical technique, bilaterally (Fig. 2).

The uterus was then placed into the abdominal cavity (warm ischemic time was set to begin at this moment) and both external iliac veins and arteries were sequentially fully clamped. A venotomy on each external iliac vein was performed and the vessels were washed with heparinized solution. Bilateral end-to-side anastomoses between the ovarian and external iliac veins were sequentially performed with running polypropylene 7-0. Every time that an anastomosis was completed, a bulldog clamp was placed on the ovarian veins in order to allow venous flow from the pelvis and lower limbs. Hemostasis control of one anastomosis at a time was performed before accomplishing arterial anastomosis. Arteriotomy with a $4 \mathrm{~mm}$ punch was then performed on the external iliac arteries; the vessels were

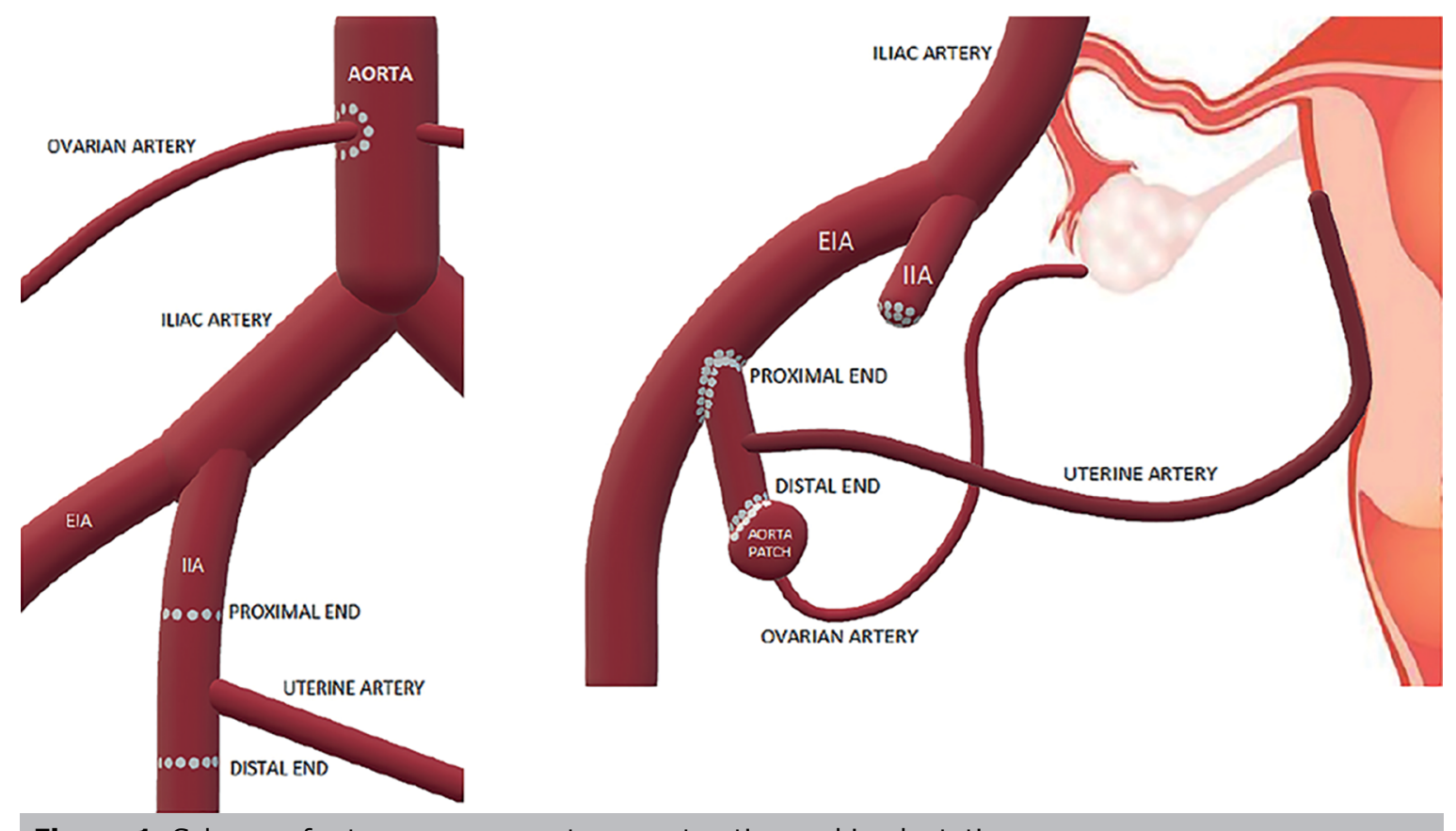

Figure 1. Scheme of artery procurement, reconstruction and implantation. 


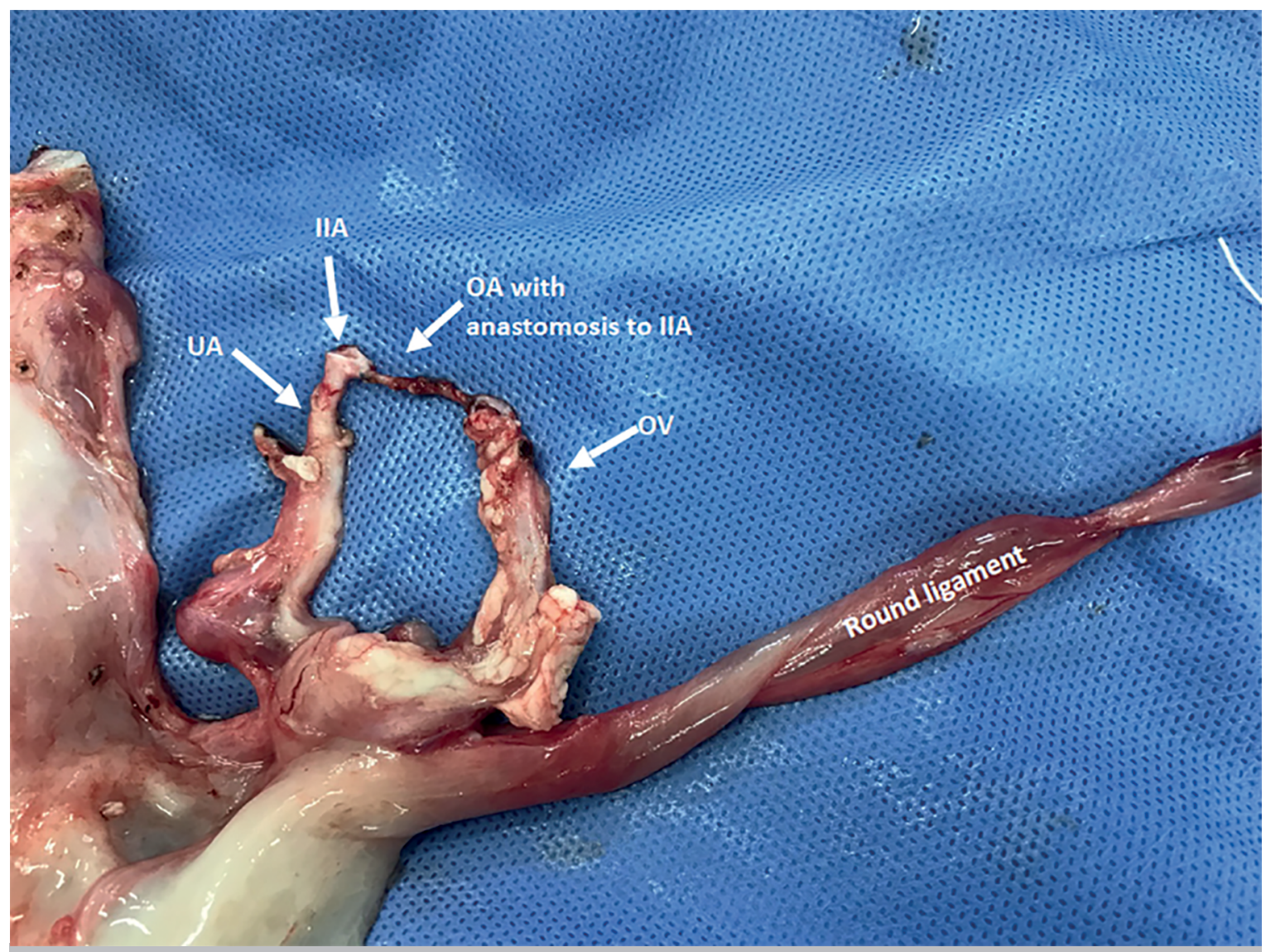

Figure 2. Back table uterus reconstruction IIA: internal iliac artery; UA: uterine artery; OA: ovarian artery; OV: ovarian vein.

washed with heparinized solution, followed by anastomosis of the proximal end of the internal iliac artery (which in turn had the uterine and ovarian arteries reconstructed into one vessel). A bulldog clamp was placed on the uterine arteries each time an anastomosis was completed in order to allow arterial flow to the pelvis and lower limbs, and hemostasis control of one anastomosis at a time was performed. Venous bulldog clamps were released and hemostasis controlled, followed by arterial clamps release (they were removed at the end of both, cold and warm ischemic times, respectively) (Fig. 3)

Rapid recovery of the uterus characteristic color with both a palpable thrill and evident pulsatility of the arteries were considered favorable clinical signs of vessel anastomosis patency (Fig. 4). Hemostasis was achieved one more time and vagino-vaginal anastomosis with running 2-0 polyglactin in one plane was performed. The uterus was fixed to the abdominal wall by re-suturing the round ligaments and the surrounding peritoneum.

Hemostasis, uterus vitality, vessels anastomosis patency and gauze, as well as instrument counts were double checked before closing the abdominal aponeurosis with running 1-0 polypropylene and skin with running 3-0 polypropylene.

One week after transplantation, uterus vitality and arterial perfusion were assessed based on direct visualization with a vaginal speculum and transvaginal Doppler ultrasound (Fig. 5). All animals underwent exploratory laparoscopies to check uterus vitality three months after transplantation.

\section{RESULTS}

We performed four autologous transplants. All animals had good response to anesthesia and surgery, without complications. All physiological variables remained within normal range during and after surgery. One of the animals developed an incisional hernia a few weeks after the procedure. All ewes were alive more than a year after transplantation. Further details are shown in Table 1.

\section{DISCUSSION}

This paper presents the first experimental UT procedures performed in domestic animals in Argentina. Different easily accessible domestic animal species have been used for UT for their similar organ sizes compared to humans (including vessels). Among these, pigs and sheep have been the most widely used in experimental UT, with two important drawbacks: they have bicornuate uteri and there are no inbred syngeneic strains, thus requiring immunosuppression in cases of allogeneic UT. Given this last issue, we chose to perform autologous transplantations on our series, the most frequently used model for UT in domestic animals (Andraus et al., 2017; Ramirez et al., 2008; Wei et al., 2013; Wranning et al., 2008).

Autologous sheep UT has been extensively used and proven to be a better model compared to pigs, since the uterus has a comparatively much smaller size and the pelvic vasculature is large, with dimensions similar to those of humans (Dahm-Kähler et al., 2008). In this regard, autologous sheep UT has been suggested as the main model to train surgical teams on human UT (Solomonov et al., 


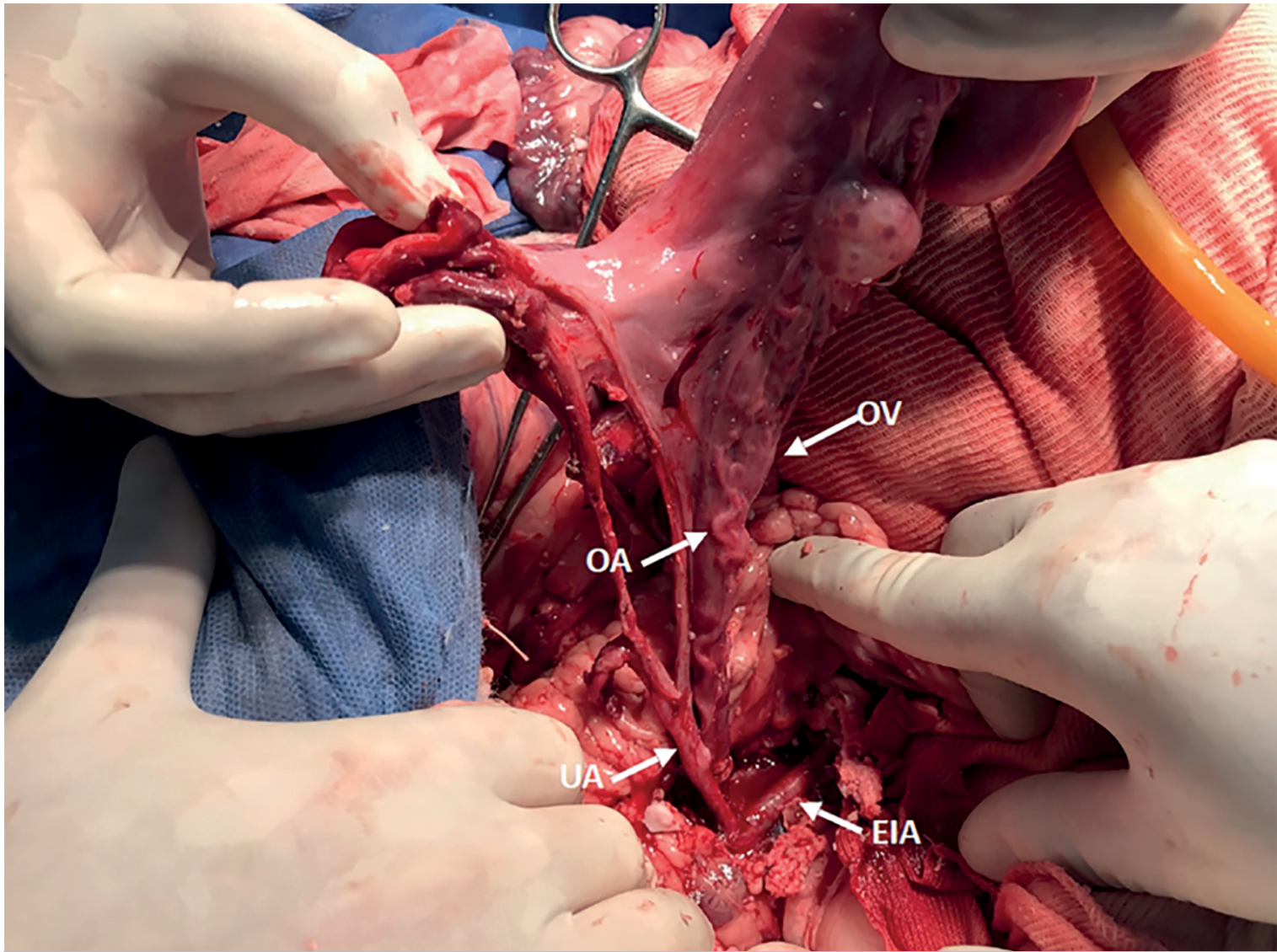

Figure 3. Implanted uterus with arterial reconstruction technique reference UA: uterine artery; OA: ovarian artery; OV: ovarian vein; EIA: external iliac artery.

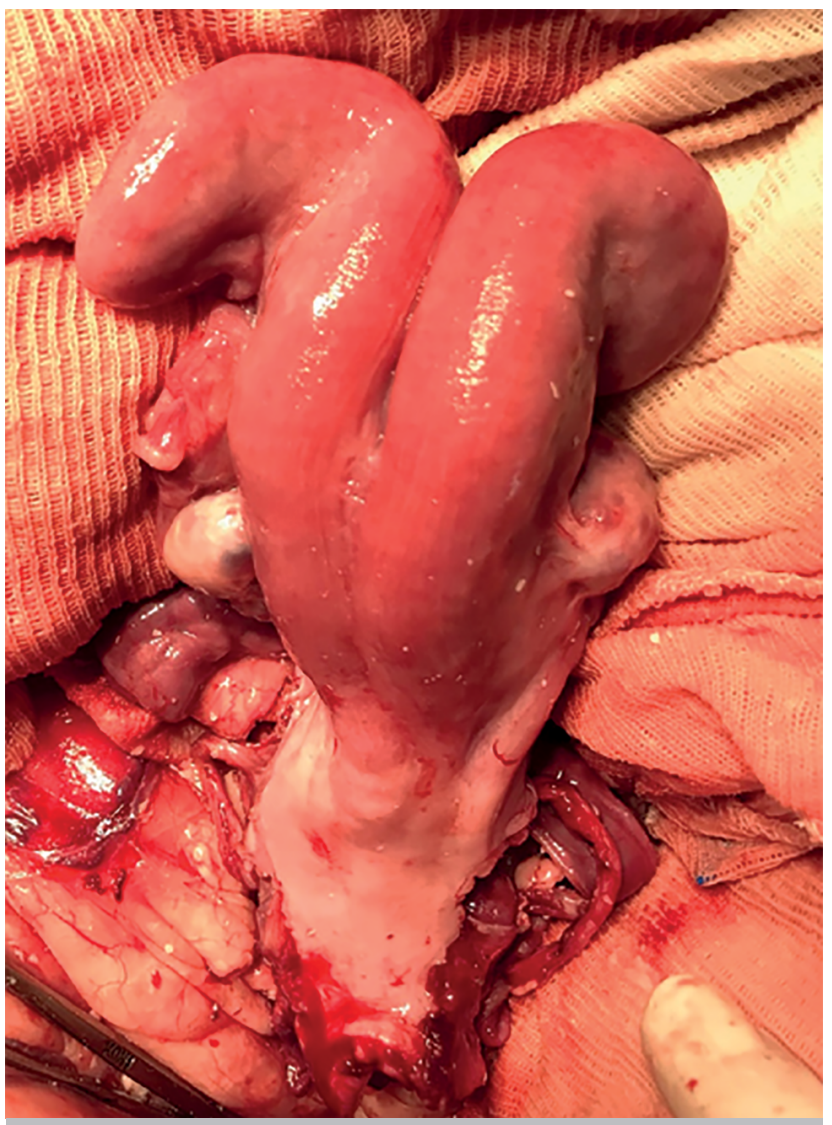

Figure 4. Reperfused uterus.

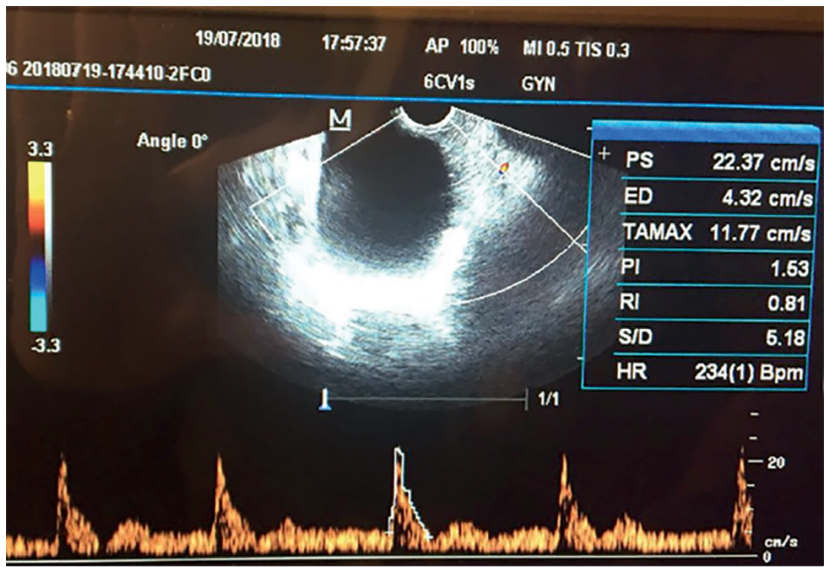

Figure 5. Transvaginal ultrasound with pulsed Doppler of the uterine artery showing adequate uterine perfusion one week after surgery PI 1.53 ; RI 0.81 .

2017). This model is technically similar to a living donor (LD) regarding shortness of vessels. It has been suggested that initially it would not be necessary to study the animal after recovery from anesthesia, with focus residing mainly on the technical aspects of organ recovery and reperfusion. Eventually, as surgical teams become more experienced, the experiment might be extended to assess graft survival after several weeks with a small laparotomy. A group from Sao Paulo (Brazil) performed total UT on a sheep model without implantation of ovarian arteries, but they euthanized the animals once surgery was concluded (Andraus et al., 2017). We conducted our experiment beyond surgery 


\begin{tabular}{|l|c|c|c|c|}
\hline \multicolumn{2}{|l|}{ Table 1. Animal and surgery data. } \\
\hline Case number & Weight (Kg) & Surgical procedure length (min) & Cold ischemic time (min) & Warm ischemic time (min) \\
\hline 1 & 55 & 315 & 105 & 65 \\
\hline 2 & 70 & 370 & 100 & 50 \\
\hline 3 & 60 & 250 & 110 & 50 \\
\hline 4 & 65 & 310 & 85 & 45 \\
\hline
\end{tabular}

to check on the patency of uterine vessels and vitality of the uterus in the mid-term, thus ensuring the success of the surgical procedure. Moreover, in order to further check on the long-term vitality of the transplanted uteri, we performed an exploratory laparoscopy on the animals three months after transplantation.

The model of LD for experimental UT in sheep developed by the Gothenburg group consisted of the excision of one uterine horn; vascular anastomosis was performed with a unilateral vascular pedicle of the anterior branch of the internal iliac artery and the complete ovarian vein, anastomosed to the ipsilateral external iliac vessels (Dahm-Kähler et al., 2008). The technique was later developed to include the ovarian artery, thus allowing the uterus to function and attain fertility (Wranning et al., 2010). In this model, an aortic patch in the origin of the ovarian artery was included and two arterial (ovarian and uterine arteries) and one venous anastomoses had to be performed. We decided to apply this technique with the entire uterus and a technical variant, performing an anastomosis between a patch of the internal iliac and ovarian arteries on bench surgery with microsurgical technique, thus simplifying the implantation of the whole uterus, with only one arterial and one venous anastomosis on each side.

\section{CONCLUSION}

Our study provided a novel surgical technique for autologous uterus transplantation in sheep described for the first time in Latin America, which yielded long-term animal survival and uterus vitality. We intend to further develop our experimental work by attempting sheep pregnancy and developing a UT protocol for human patients.

\section{CONFLICT OF INTEREST}

The authors have no conflicts of interest to declare.

\section{Corresponding author:}

Martín A Maraschio

Liver, Kidney and Pancreas Transplant Program

Hospital Privado Universitario

Córdoba, Argentina.

E-mail: mmaraschio@gmail.com

\section{REFERENCES}

Andraus W, Ejzenberg D, Dos Santos RM, Mendes LR, Arantes RM, Baracat EC, D'Albuquerque LA. Sheep Model for Uterine Transplantation: The Best Option Before Starting a Human Program. Clinics. 2017;72:178-82. PMID: 28355364 DOI: $10.6061 /$ clinics/2017(03)08

Beski S, Gorgy A, Venkat G, Craft IL, Edmonds K. Gestational surrogacy: a feasible option for patients with Rokitansky syndrome. Hum Reprod. 2000;15:2326-8. PMID: 11056126 DOI: $10.1093 /$ humrep/15.11.2326

Brännström M. Uterus transplantation and beyond. J Mater Sci Mater Med. 2017;28:70. PMID: 28357688 DOI: $10.1007 / \mathrm{s} 10856-017-5872-0$
Brännström M, ed. Uterus Transplantation. Cham: Springer International Publishing; 2020.

Brännström M, Diaz-Garcia C, Hanafy A, Olausson M, Tzakis A. Uterus transplantation: animal research and human possibilities. Fertil Steril. 2012;97:1269-76. PMID: 22542990 DOI: $10.1016 /$ j.fertnstert.2012.04.001

Brännström $M$, Johannesson $L$, Dahm-Kähler $P$, Enskog $A$, Mölne J, Kvarnström N, Diaz-Garcia C, Hanafy A, Lundmark C, Marcickiewicz J, Gäbel M, Groth K, Akouri R, Eklind S, Holgersson J, Tzakis A, Olausson M. First clinical uterus transplantation trial: a six-month report. Fertil Steril. 2014;101:1228-36. PMID: 24582522 DOI: 10.1016/j.fertnstert.2014.02.024

Brännström $M$, Johannesson $L$, Bokström $H$, Kvarnström $N$, Mölne J, Dahm-Kähler P, Enskog A, Milenkovic M, Ekberg J, Diaz-Garcia C, Gäbel M, Hanafy A, Hagberg H, Olausson M, Nilsson L. Livebirth after uterus transplantation. Lancet. 2015;385:60716. PMID: 25301505 DOI: 10.1016/S0140-6736(14)61728-1

Brännström M, Enskog A, Kvarnström N, Ayoubi JM, DahmKähler P. Global results of human uterus transplantation and strategies for pre-transplantation screening of donors. Fertil Steril. 2019;112:3-10. PMID: 31277765 DOI: 10.1016/j.fertnstert.2019.05.030

Castellón LAR, Amador MIG, González RED, Sarmiento JEM, Díaz-García C, Kvarnström N, Brännström M. The history behind successful uterine transplantation in humans. JBRA Assist Reprod. 2017; 21:126-34. PMID: 28609280 DOI: $10.5935 / 1518-0557.20170028$

Dahm-Kähler $P$, Wranning $C$, Lundmark $C$, Enskog A, Mölne J, Marcickiewicz J, El-Akouri RR, McCracken J, Brännström M. Transplantation of the uterus in sheep: methodology and early reperfusion events. J Obstet Gynaecol Res. 2008;34:784-93. PMID: 18834335 DOI: $10.1111 / \mathrm{j} .1447-0756.2008 .00854 . x$

Dahm-Kähler P, Diaz-Garcia C, Brännström M. Human uterus transplantation in focus. Br Med Bull. 2016;117:6978. PMID: 26888381 DOI: $10.1093 / \mathrm{bmb} / \mathrm{ldw} 002$

Daolio J, Palomba S, Paganelli S, Falbo A, Aguzzoli L. Uterine transplantation and IVF for congenital or acquired uterine factor infertility: A systematic review of safety and efficacy outcomes in the first 52 recipients. PLoS One. 2020;15:e0232323. PMID: 32348371 DOI: 10.1371/journal.pone.0232323

Farrell RM, Falcone T. Uterus transplant: new medical and ethical considerations. Lancet. 2015;385:581-2. PMID: 25303786 DOI: $10.1016 /$ S0140-6736(14)61792-X

Friedler S, Grin L, Liberti G, Saar-Ryss B, Rabinson Y, Meltzer S. The reproductive potential of patients with Mayer-Rokitansky-Küster-Hauser syndrome using gestational surrogacy: a systematic review. Reprod Biomed Online. 2016;32:54-61. PMID: 26626805 DOI: 10.1016/j. rbmo.2015.09.006 
Practice Committee of the American Society for Reproductive Medicine. American Society for Reproductive Medicine position statement on uterus transplantation: a committee opinion. Fertil Steril. 2018;110:605-10. PMID: 30196945 DOI: $10.1016 /$ j.fertnstert.2018.06.017

Racho El-Akouri R, Kurlberg G, Brännström M. Successful uterine transplantation in the mouse: pregnancy and postnatal development of offspring. Hum Reprod. 2003;18:2018-23. PMID: 14507815 DOI: 10.1093/humrep/deg396

Ramirez ER, Ramirez DK, Pillari VT, Vasquez $\mathrm{H}$, Ramirez $\mathrm{HA}$. Modified uterine transplant procedure in the sheep model. J Minim Invasive Gynecol. 2008;3:311-4. PMID: 18439503 DOI: $10.1016 /$ j.jmig.2008.01.014

Riggan KA, Khan Z, Langstraat CL, Allyse MA. Provider Knowledge and Support of Uterus Transplantation: Surveying Multidisciplinary Team Members. Mayo Clin Proc Innov Qual Outcomes. 2020;4:150-8. PMID: 32280925 DOI: 10.1016/j.mayocpiqo.2019.11.001
Solomonov E, Marcus Braun N, Siman-Tov Y, Ben-Shachar I. Team preparation for human uterus transplantation: Autologous transplantation in sheep model. Clin Transplant. 2017;31:e13137. PMID: 29032587 DOI: 10.1111/ctr.13137

Wei L, Xue T, Yang $H$, Zhao GY, Zhang G, Lu ZH, Huang YH, Ma XD, Liu HX, Liang SR, Yang F, Chen BL. Modified uterine allotransplantation and immunosuppression procedure in the sheep model. PLoS One. 2013;8:e81300. PMID: 24278415 DOI: $10.1371 /$ journal.pone.0081300

Wranning CA, Akhi SN, Kurlberg G, Brännström M. Uterus transplantation in the rat: model development, surgical learning and morphological evaluation of healing. Acta Obstet Gynecol Scand. 2008;87:1239-47. PMID: 18951268 DOI: $10.1080 / 00016340802484966$

Wranning CA, Marcickiewicz J, Enskog A, Dahm-Kähler P, Hanafy A, Brännström M. Fertility after autologous ovine-tubal-ovarian transplantation by vascular anastomosis to the external iliac vessels. Hum Reprod. 2010;25:1973-9. PMID: 20519245 DOI: 10.1093/humrep/deq130 